\section{How successful are weight management programmes in obese cats?}

\section{Erin O'Connell', Maria Williams', Shelley Holden', Vincent Biourge ${ }^{2}$, Alexander German'}

1 University of Liverpool, Cheshire, UK, 2Royal Canin Research Center, Aimargues, France

The most common approach for controlled weight loss in cats is dietary caloric restriction, using a purpose-formulated diet. However, most previous studies have only assessed short-terms outcomes (i.e. from the first 2-3 months of weight loss), and no previous study has examined overall success (i.e. likelihood of reaching target weight). The aim of this study was to determine outcome of obese cats undergoing weight management, and to determine what factors are associated with overall success.

The data from records of obese cats attending the Royal Canin Weight Management Clinic, University of Liverpool, were reviewed, and were eligible for inclusion if there was a known outcome for weight loss (i.e. the cat had completed or stopped their programme). Cats were classed as "completed" when they reached target weight, "stopped prematurely" when their programme was terminated before reaching target weight, and "died" when the cat had been euthanased before reaching target weight. Simple and multiple logistic regression analysis were used to identify factors associated with the likelihood of completing the weight loss programme.

A total of 62 cats were ultimately included, and $28(45 \%)$ completed the programme. The remaining 32 cats (55\%) did not reach target weight, of which 2 (3\%) were euthanased for unrelated reasons. Reasons for cats stopping the programme prematurely included inability to contact owner (19), the owner requested that the programme be completed prior to reaching target weight (5), the cat developed another illness (3), refusal to comply with requirements for weight management (2), owner illness (2), personal issues of the owner (1). Simple and multiple logistic regression analysis revealed that cats with a greater starting body fat were less likely to complete $(P=0.02)$, whilst those that lost weight more rapidly were more successful $(P<0.001)$.

This study highlights the fact that the most obese cats are more likely to fail on a weight programme. Further investigations are now required to better clarify the reasons for success and failure in individual cases.

\section{Management decisions in a cohort of UK pet kittens, and changes made up to 18 months of age}

\section{Claire Roberts, Timothy Gruffydd-Jones, Jane Murray}

School of Veterinary Sciences, University of Bristol, Bristol, UK

Certain decisions by owners in the management of their cats, including outdoor access, living with other cats and vaccinations can impact on health, such as lower urinary tract disorders, road traffic accidents and infectious diseases. However, little information exists on when these decisions are made and the extent to which they change during a cat's early life. The aim of this study was to investigate changes made to these variables prior to age 18 months.

The Bristol Cats Study is a long-term longitudinal study involving data collection by owner-completed questionnaires. Pertinent data were used from the first four questionnaires (ages 2-4 months, six months, 12 months and 18 months). Only cats with all four questionnaires returned were included in this study $(\mathrm{n}=1422)$.

For most cats $(80.7 \%)$ there was no change in whether they had outdoor access between six and 18 months; $25.4 \%$ were indoors only or had restricted lead/run access outdoors and $55.2 \%$ had indoor and outdoor access. Some were 'indoor only' cats or had lead/run access at six months, but allowed outdoors by 12 months (11.5\%) or 18 months (3.0\%). A further $4.5 \%$ were allowed outdoors at some point but subsequently had outdoor access restricted.

The presence/absence of other cats in the household tended to be consistent from age 2-4 months to 18 months, (76.5\% in multi-cat households; $16.6 \%$ in single-cat households). Two percent began in multi-cat households but became 'single cats' and remained so, whereas $4.2 \%$ were initially alone but were joined by one or more cats. The number of cats in 'multi-cat' households and changes other than to the overall number was not investigated.

Vaccination data revealed 1324/1404 cats (94.3\%) received their two primary vaccines by six months of age. Only 743 (56.1\%) of these received their third vaccination a year later.

Relatively few changes occurred during the first 18 months with regards to outdoor access and single/multi-cat households. The lifestyle decisions made at an early age tend to remain consistent to 18 months. It is important for veterinarians to discuss these factors and their implications on health with owners at their first appointment. The suggestion that many cats start but do not complete the initial vaccine course with the first annual vaccine warrants further investigation. 